\title{
Fluoride toothpaste prevents caries in children and adolescents at fluoride concentrations of 1000 ppm and above
}

\author{
Abstracted from \\ Cochrane Database Syst Rev 2010; issue 1

\section{Question: What is the relative effectiveness of fluoride toothpastes of different concentrations in preventing dental caries} \\ in children and adolescents?
}

Walsh T, Worthington HV, Glenny AM, Appelbe P, Marinho VCC, Shi X.

Fluoride toothpastes of different concentrations for preventing dental caries in children and adolescents.

Address for correspondence: Luisa Fernandez Mauleffinch, Review Group Co-ordinator, Cochrane Oral Health Group, MANDEC, School of Dentistry, University of Manchester, Higher Cambridge Street, Manchester, M15 6FH, UK. E-mail luisa.fernandez@manchester.ac.uk

Data sources The Cochrane Oral Health Group's Trials Register, Cochrane Central Register of Controlled Trials, MEDLINE and EMBASE databases were searched. Previously published systematic reviews of fluoride toothpastes were also screened to identify any reports that met the inclusion criteria. In addition, the trials database at www. clinicaltrials.gov and the metaRegister of Controlled Trials (www. controlled-trials.com) were searched to identify any ongoing studies of relevance.

Study selection Randomised controlled trials (RCT) and cluster-RCT that compared fluoride toothpaste with placebo or fluoride toothpaste of a different concentration in children of up to 16 years of age, with a followup period of at least 1 year, were included.

Data extraction and synthesis Inclusion of studies, data extraction and quality assessment were undertaken independently and in duplicate by two members of the review team. Disagreements were resolved by discussion and consensus or by a third party. The primary effect measure was the prevented fraction (PF, the caries increment of the control group minus the caries increment of the treatment group, expressed as a proportion of the caries increment in the control group). Where it was appropriate to pool data, network meta-analysis, network meta-regression or meta-analysis models were used. Potential sources of heterogeneity were specified a priori and examined through random-effects metaregression analysis where appropriate.

Results From 535 studies found, 75 were included. From these, 71 studies (79 trials) contributed data to the network meta-analysis, network metaregression or meta-analysis. For the 66 studies (74 trials) that contributed to the network meta-analysis of decayed, missing or filled surfaces $[D(M) F S]$ in the mixed or permanent dentition, the caries preventive effect of fluoride toothpaste increased significantly with higher fluoride concentrations, with a D(M)FS PF compared with placebo of $23 \%$ [95\% credible interval (Crl), 19-27\%] for 1000/ 1055/ $1100 / 1250$ ppm concentrations, rising to $36 \%$ (95\% Crl, 27-44\%)

This paper is based on a Cochrane Review published in the Cochrane Library 2010, issue 1 (see www.thecochranelibrary.com for information). Cochrane Reviews are regularly updated as new evidence emerges and in response to feedback, and the Cochrane Library should be consulted for the most recent version of the review. for toothpastes with a concentration of 2400/ 2500/ 2800 ppm. Concentrations of 440/500/ $550 \mathrm{ppm}$ and below showed no statistically significant effect when compared to placebo.

There is some evidence of a dose-response relationship in that the $\mathrm{PF}$ increased as the fluoride concentration increased from the baseline although this was not always statistically significant. The effect of fluoride toothpaste also increased with baseline level of D(M)FS and supervised brushing, though this did not reach statistical significance. Six studies assessed the effects of fluoride concentrations on the deciduous dentition with equivocal results dependent upon the fluoride concentrations compared and the outcome measure. Compliance with treatment regimen and unwanted effects was assessed in only a minority of studies. When reported, no differential compliance was observed and unwanted effects such as soft tissue damage and tooth staining were minimal.

Conclusions This review confirms the benefits of using fluoride toothpaste in preventing caries in children and adolescents compared with placebo, but only statistically significantly at fluoride concentrations of $1000 \mathrm{ppm}$ and above. The relative caries preventive effects of fluoride toothpastes of different concentrations increase with higher fluoride concentration. The decision of what fluoride levels to use for children aged under 6 years should be balanced with the risk of fluorosis.

\section{Commentary}

This Cochrane Review is part of a series of systematic reviews on interventions with topical fluorides and addresses an issue that is of concern to dentists currently, and indeed has been for some time, namely, caries prevention in children and adolescents.

The ability of fluorides to prevent dental demineralisation has been well known and documented during the last 80 years. Since Arnold et al. ${ }^{1}$ conducted a clinical study about fluoride supplements in 1960 a lot of papers about this subject have been published. Doing a simple search at Pubmed with the keywords "Dental Caries/ prevention and control"[Mesh] AND "Fluorides"[Mesh], limit 0-18 years, identifies 2276 articles. The systematic reviews only consider the studies with high methodological quality, to reduce bias and allow the dentist to trust in the results. Surely, the first work done by Arnold et al. wouldn't be included there was no control group for comparison — its results are probably overvalued. ${ }^{1}$

Despite this well developed research area, caries is still a common problem that affects the quality of life in the youngest population. Oral health is one of the topics focused on by the World Health 
Organization, which aims to improve oral health outcomes of the world population ${ }^{2}$ by increasing the proportion of children free of caries at 6 years old and reducing the proportion of decayed, missing or filled teeth at 12 years, among other targets. To achieve these goals, the decision-makers need reliable information about the available interventions. Fluoride toothpaste seems to be a cost-effective intervention in preventing dental caries. This review deals with one of the parts of this equation: the effectiveness of different concentrations of fluoride.

The strengths of this review are many. Following the strict concepts of the Cochrane Collaboration, four reviewers worked independently in identifying and appraising the included studies and extracting the data. This is one of the effective methodological tools that reduce bias in the review. The searches made for the RCT were also exhaustive, with no limit on language, and reviewers contacting the authors when it was necessary and even searching for ongoing trials. This review is therefore robust in its methods.

Among the weaknesses, we should consider how only $10 \%$ of the included studies describe clearly how the randomisation sequence was concealed. It is also difficult to assess the level of risk of attrition bias, as insufficient information was available in the original studies. The average dropout ranges from $4 \%$ at year 2 to $66 \%$ at year 3 of studies. Even the allocation was unclear in most of the studies, although the baseline characteristics seemed to be well balanced, which is very important when comparing the effect of interventions between groups. The blinding was well-documented: in $92 \%$ of the trials, both participants and clinical examiners were blinded to the allocated intervention. Considering all these characteristics, it is possible to determine that the review has a good internal validity and the results are reliable.

The included studies range in publication date from 1955 to 2008 . This raises the issue of variation in the criteria used to define caries. As since following the National Institutes of Health Consensus
Development Conference in $2001^{3}$ there has been an increasing focus on early demineralisation or white spot lesions as an indicator of need for preventive and therapeutic interventions.. However, this is addresses by the use of PF primary effect measure.

The calculations of a caries preventive effect of $23 \%$ (95\% CrI, 19-27\%) for 1000-1250 ppm concentrations and 36\% (95\% CrI, $27-44 \%)$ for concentrations of $2400-2800 \mathrm{ppm}$ are results with important clinical significance. The recommendation to use fluoride toothpaste to prevent caries in children and adolescents is supported by these results after considering the risk of fluorosis. Based on the presented evidence, clinicians and public health decisionmakers should consider fluoride toothpastes as a useful tool in caries prevention.

Graciela Rasines

Evidence-based Dentistry Commission, Dental Association of Argentina, Buenos Aires, Argentina

\section{Practice point}

- This review confirms that toothpastes containing at least 1000 parts ppm fluoride are effective at preventing tooth decay in children. The decision of what fluoride levels to use for children under 6 years should be balanced with the risk of fluorosis.

1. Arnold FA, McClure FJ, White CL. The Caries Status of Children Who Ingested Sodium Fluoride Tablets. Dental Progress 1960 1: 12-16.

2. Petersen PE. Priorities for research for oral health in the 21 st Century - the approach of the WHO Global Oral Health Programme. Community Dent Health 2005; 22: $71-74$.

3. National Institute of Health Consensus Development Panel. National Institutes of Health Consensus Development Conference statement. Diagnosis and management of dental caries throughout life, March 26-28, 2001. I Am Dent Assoc. 2001; 132: $1153-1161$.

Evidence-Based Dentistry (2010) 11, 6-7. doi:10.1038/sj.ebd.6400698 